Document downloaded from:

http://hdl.handle.net/10251/37911

This paper must be cited as:

Angosto Hernández, C.; Kakol, J.; Kubzdela, A.; López Pellicer, M. (2013). A quantitative version of Krein's theorems for Fréchet spaces. Archiv der Mathematik. 101(1):65-77. doi:10.1007/s00013-013-0513-4.

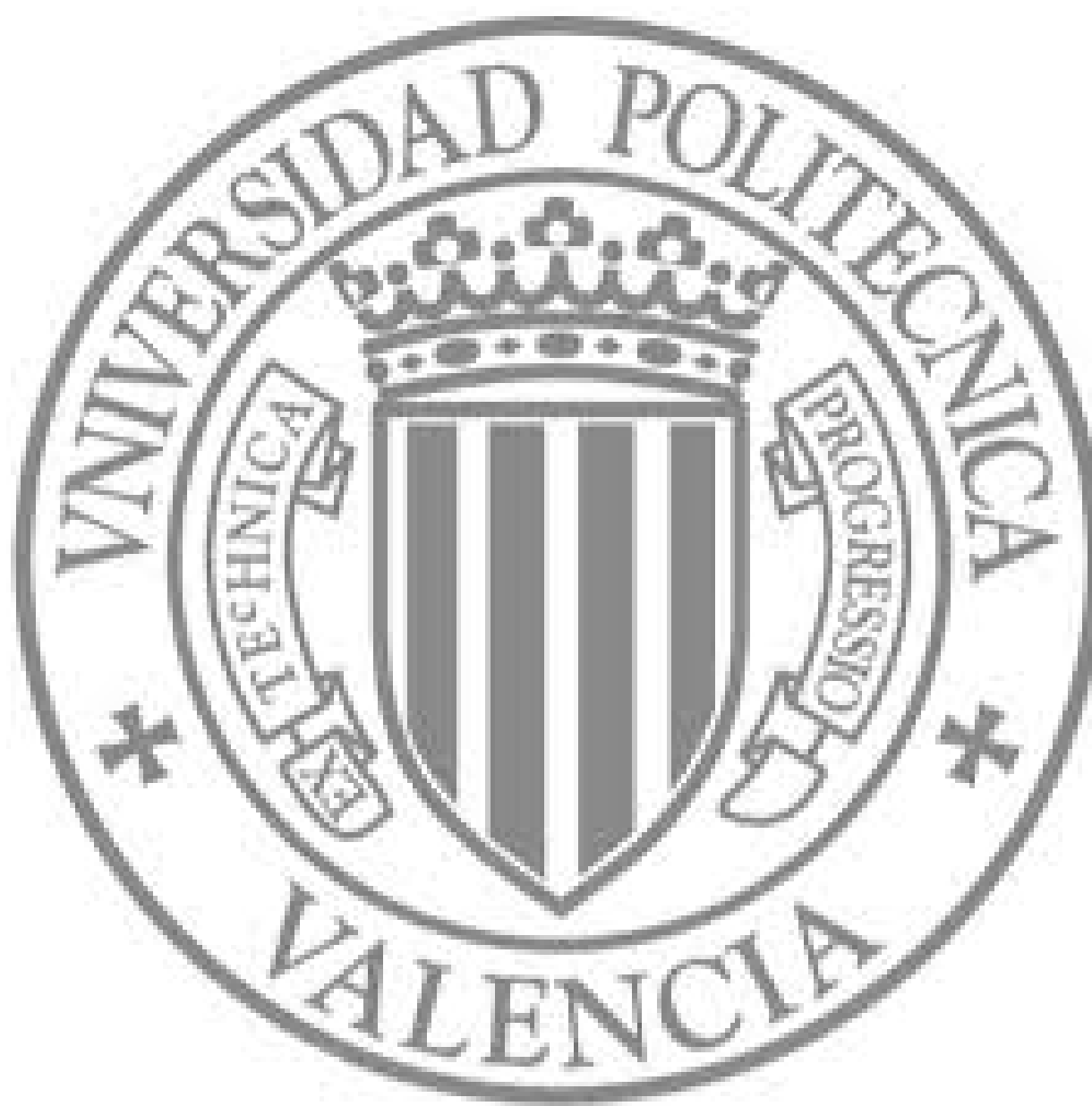

The final publication is available at

http://link.springer.com/content/pdf/10.1007\%2Fs00013-013-0513-4.pdf

Copyright Springer Verlag (Germany) 


\title{
A quantitative version of Krein's theorems for Fréchet spaces
}

\author{
Carlos Angosto, Jerzy Kạkol, Albert Kubzdela and Manuel \\ López-Pellicer
}

\begin{abstract}
For a Banach space $E$ and its bidual space $E^{\prime \prime}$ the following function $k(H):=\sup _{y \in \bar{H}^{\sigma\left(E^{\prime \prime}, E^{\prime}\right)}} \inf _{x \in E}\|y-x\|$ defined on bounded subsets $H$ of $E$ measures how far $H$ is from being $\sigma\left(E, E^{\prime}\right)$-relatively compact in $E$. This concept, introduced independently by Granero (2006) and Cascales-Marciszewski-Raja (2006), has been used to study a quantitative version of Krein's theorem for Banach spaces $E$ and spaces $C_{p}(K)$ over compact $K$. In the present paper a quantitative version of Krein's theorem on convex envelopes $c o H$ of weakly compact sets $H$ is proved for Fréchet spaces, i.e. metrizable and complete locally convex spaces. For a Fréchet space $E$ the above function $k(H)$ reads as follows $k(H):=\sup \left\{d(h, E): h \in \bar{H}^{\sigma\left(E^{\prime \prime}, E^{\prime}\right)}\right\}$, where $d(h, E)$ is the natural distance of $h$ to $E$ in the bidual $E^{\prime \prime}$. The main result of the paper is the following

Theorem: For a bounded set $H$ in a Fréchet space $E$ the following inequality holds $k(\mathrm{coH})<\left(2^{n+1}-2\right) k(H)+\frac{1}{2^{n}}$ for all $n \in \mathbb{N}$. Consequently this yields also the following formula $k(\mathrm{coH}) \leq \sqrt{k(H)}(3-$ $2 \sqrt{k(H)})$.

Hence $c o H$ is weakly relatively compact provided $H$ is weakly relatively compact in $E$. This extends a quantitative version of Krein's theorem for Banach spaces (obtained by Fabian, Hajek, Montesinos, Zizler, Cascales, Marciszewski and Raja) to the class of Fréchet space. We also define and discuss two another measures of weak non-compactness $l k(H)$ and $k^{\prime}(H)$ for a Fréchet space and provide two quantitative versions of Krein's theorem for the both functions.
\end{abstract}

Mathematics Subject Classification (2010). Primary 46A50; Secondary $54 \mathrm{C} 35$.

Keywords. Krein's theorem, Compactness, Fréchet space, Space of continuous functions.

The research was supported for the first named author by the project MTM2008-05396 of the Spanish Ministry of Science and Innovation, for the second named author by National Center of Science, Poland, grant no. N N201 605340. 


\section{Introduction}

In the last decade several quantitative counterparts of some other classical results (including Gantmacher, Eberlein-Grothendieck, Grothendieck, KreinSmulyan' theorems) have been proved by several specialists, see for example $[1],[2],[3],[4],[5],[8],[10],[11]$ and references. It turns out that these new versions strengthen the original results and provide new applications both in functional analysis and topology.

The classical Krein's theorem for Banach spaces $E$ states that for a weakly relatively compact set $K$ in $E$ its closed convex envelop is weakly compact, i.e. compact in the weak topology $\sigma\left(E, E^{\prime}\right)$ of $E$, see for example [8, Theorem 3.5.8]. The following question which refers to this theorem was formulated in $[9]$ :

(*) Let $H$ be a bounded set in a Banach space $E$ and let $B_{E^{\prime \prime}}$ be the closed unit ball in the bidual $E^{\prime \prime}$ of $E$. Assume that $H$ is a $\varepsilon$-weakly relatively compact set (in short $\varepsilon-W R K$ ), i.e. $\bar{H}^{\sigma\left(E^{\prime \prime}, E^{\prime}\right)} \subset E+\varepsilon B_{E^{\prime \prime}}$ for some $\varepsilon \geq 0$. Does the same hold for its convex envelope $c o H$ ?

Clearly the answer is positive if $\varepsilon=0$, which is the statement of the Krein's theorem. As mentioned in [9], problem $\left(^{*}\right)$ was motivated by some results about closed subspaces of Weakly Compactly Generated Banach spaces, see [9, Theorem 15]. This, and the fact that the class of Weakly Compactly Generated Fréchet spaces is sufficiently large and attracted also specialists, see for example results of Khurana [13], motivate also the present work.

In $[9$, Theorem 2] it was proved by applying Ptak's combinatorial lemma, that whenever $H$ is $\varepsilon-W R K$ for some $\varepsilon>0$, then $c o H$ is $2 \varepsilon-W R K$. Moreover if $B_{E^{\prime \prime}}$ is $\sigma\left(E^{\prime \prime}, E^{\prime}\right)$-angelic, then $c o H$ is $\varepsilon-W R K$. The latter result applies to separable Banach spaces, or more generally, Weakly Compactly Generated Banach spaces or even Weakly Lindelöf Determined Banach spaces, see again [8].

In [7, Corollary 3.4] Cascales, Marciszewski and Raja obtained more general theorem stating that for a compact space $K$ and uniformly bounded $H \subset C(K)$ the following evaluation holds

$$
\hat{d}\left(\overline{c o H}^{\mathbb{R}^{K}}, C(K)\right) \leq 2 \hat{d}\left(\bar{H}^{\mathbb{R}^{K}}, C(K)\right),
$$

where $\hat{d}$ is the Hausdorff non-symmetrized distance.

Let $E$ be a Banach space and let $E^{\prime \prime}$ be its bidual. Following [7] and [10] define the function

$$
k(H):=\sup _{y \in \bar{H}^{\sigma\left(E^{\prime \prime}, E^{\prime}\right)}} \inf _{x \in E}\|y-x\|
$$

for any bounded set $H$ in $E$. Clearly $k(H)$ measures how far $H$ is from being weakly relatively compact in $E$. The above result from [7, Corollary 3.4] implies that $k(\mathrm{coH}) \leq 2 k(H)$ for any bounded set $H$ in a Banach space $E$, see also [1]. Note that the equality $k(c o H)=k(H)$ fails in general, see [10], $[11]$. 
In the present paper we continue this line of research for the class of Fréchet spaces. The main result as stated in Abstract implies that if $H$ is a bounded set in a Fréchet space $E$ and if $k(H)<\varepsilon^{2}$, then $k(c o H)<\varepsilon(3-2 \varepsilon)$ for each $\varepsilon>0$, where the function $k(H)$ for the case $E$ being a Fréchet space takes the form as mentioned in Abstract, see also below.

Let $E$ be a Fréchet space and let $\left(U_{n}\right)_{n}$ be the family of absolutely convex neighbourhoods of zero such that $\left(\frac{1}{n} U_{n}\right)_{n}$ is a basis of neighbourhoods of zero. By $\left(E^{\prime}, \beta\left(E^{\prime}, E\right)\right)$ and $\left(E^{\prime \prime}, \beta\left(E^{\prime \prime}, E^{\prime}\right)\right)$ we mean the strong dual of $E$ and $\left(E^{\prime}, \beta\left(E^{\prime}, E\right)\right)$, respectively. By $\|h\|_{n}=\sup \left\{|h(u)|: u \in U_{n}^{0}\right\}$ we denote the seminorm in $E^{\prime \prime}$ associated with $U_{n}^{0}$ and $d_{n}$ means the pseudometric defined by $\|\cdot\|_{n}$. The restriction of $\|\cdot\|_{n}$ to $E$, also denoted by $\|\cdot\|_{n}$, is the seminorm defined by $U_{n}$. The topology of $E$ can be defined by the $F$-norm

$$
d(x, y):=\sum_{n} 2^{-n}\|x-y\|_{n}\left(1+\|x-y\|_{n}\right)^{-1}
$$

for $x, y \in E$. Also the topology of the space $\left(E^{\prime \prime}, \beta\left(E^{\prime \prime}, E^{\prime}\right)\right)$ is defined by the $F$-norm

$$
d\left(x^{* *}, y^{* *}\right):=\sum_{n} 2^{-n}\left\|x^{* *}-y^{* *}\right\|_{n}\left(1+\left\|x^{* *}-y^{* *}\right\|_{n}\right)^{-1}
$$

for all $x^{* *}, y^{* *} \in E^{\prime \prime}$. Additionally, without loss of generality, we will assume that $U_{n+1} \subset U_{n}$ for $n \in \mathbb{N}$; and this clearly implies that $\left\|x^{* *}\right\|_{n} \leq\left\|x^{* *}\right\|_{n+1}$ for $n \in \mathbb{N}$ and each $x^{* *} \in E^{\prime \prime}$.

\section{More about the measure of weak non-compactness $k(H)$}

By a measure of the weak non-compactness we mean a function $\mu$ defined on the family of bounded subsets of a Fréchet space $E$ such that if $A, B \subset E$ are bounded then (i) $\mu(A)=0$ if and only if $A$ is weakly relatively compact, (ii) if $A \subset B$, then $\mu(A) \leq \mu(B)$. If $H$ is a bounded subset of $E$ then $H^{0}$ is a neighbourhood of zero in $\left(E^{\prime}, \beta\left(E^{\prime}, E\right)\right)$ and the bipolar $H^{00}$ is a compact subset of $\left(E^{\prime \prime}, \sigma\left(E^{\prime \prime}, E^{\prime}\right)\right)$ which is bounded in the strong topology $\beta\left(E^{\prime \prime}, E^{\prime}\right)$. Therefore a bounded subset $H$ of $E$ is weakly relatively compact if and only if $\bar{H}^{\sigma\left(E^{\prime \prime}, E^{\prime}\right)}$ is contained in $E$. In [6] we introduced the following two functions for a Fréchet spaces $E$.

$$
\begin{aligned}
k(H) & :=\sup \left\{d(h, E): h \in \bar{H}^{\sigma\left(E^{\prime \prime}, E^{\prime}\right)}\right\}, \\
k_{n}(H) & :=\sup \left\{d_{n}(h, E): h \in \bar{H}^{\sigma\left(E^{\prime \prime}, E^{\prime}\right)}\right\} .
\end{aligned}
$$

Observe that $k(H)$ is a measure of weak non-compactness and a bounded set $H \subset E$ is weakly relatively compact if and only if $k(H)=0$ if and only if $k_{n}(H)=0$ for each $n \in \mathbb{N}$.

We need the following lemma which will be used for the proof of Theorem 3.5. 
Lemma 2.1. If $H$ is a bounded subset of a Fréchet space $E$ and $n \in \mathbb{N}$ then

$$
k(H) \leq \frac{2^{n}-1}{2^{n}} \frac{k_{n}(H)}{1+k_{n}(H)}+\frac{1}{2^{n}} .
$$

Proof. Since the function $f(x)=x /(1+x)$ is strictly increasing and $d_{m}(h, e) \leq$ $d_{n}(h, e)$ for each $h, e \in E^{\prime \prime}$ and $m \leq n$ (recall that $d_{m}(h, e) \leq d_{m+1}(h, e)$ for all $m \in \mathbb{N}$ ), we derive that

$$
\begin{aligned}
\sum_{m=1}^{\infty} \frac{1}{2^{m}} \frac{d_{m}(h, e)}{1+d_{m}(h, e)} & <\sum_{m=1}^{n} \frac{1}{2^{m}} \frac{d_{n}(h, e)}{1+d_{n}(h, e)}+\sum_{m=n+1}^{\infty} \frac{1}{2^{m}}= \\
& =\left(1-\frac{1}{2^{n}}\right) \frac{d_{n}(h, e)}{1+d_{n}(h, e)}+\frac{1}{2^{n}}= \\
& =\left(2^{n}-1\right) \frac{1}{2^{n}} \cdot \frac{d_{n}(h, e)}{1+d_{n}(h, e)}+\frac{1}{2^{n}} .
\end{aligned}
$$

Then

$$
\begin{aligned}
d(h, E) & =\inf _{e \in E} \sum_{m=1}^{\infty} \frac{1}{2^{m}} \cdot \frac{d_{m}(h, e)}{1+d_{m}(h, e)} \leq \frac{2^{n}-1}{2^{n}} \frac{\inf _{e \in E} d_{n}(h, e)}{1+\inf _{e \in E} d_{n}(h, e)}+\frac{1}{2^{n}}= \\
& =\frac{2^{n}-1}{2^{n}} \cdot \frac{d_{n}(h, E)}{1+d_{n}(h, E)}+\frac{1}{2^{n}} .
\end{aligned}
$$

Finally, if we take the supremum over all $h \in \bar{H}^{\sigma\left(E^{\prime \prime}, E^{\prime}\right)}$, we have the following

$$
k(H) \leq \frac{2^{n}-1}{2^{n}} \sup _{h \in \bar{H}^{\sigma\left(E^{\prime \prime}, E^{\prime}\right)}} \frac{d_{n}(h, E)}{1+d_{n}(h, E)}+\frac{1}{2^{n}}=\frac{2^{n}-1}{2^{n}} \frac{k_{n}(H)}{1+k_{n}(H)}+\frac{1}{2^{n}} .
$$

\section{Quantitative version of Krein's theorem}

In this section we prove a promised quantitative versions of the Krein's theorem for Fréchet spaces. Our approach will use the concept of $\varepsilon$-interchange of limits. This notion, originally introduced by Grothendieck in [12] for $\varepsilon=0$, was extended for $\varepsilon>0$ in [9]. If $\varepsilon \geq 0$ we say that $H \varepsilon$-interchanges limits with a subset $B$ of $E^{\prime}$ if

$$
\left|\lim _{p} \lim _{m} u_{p}\left(h_{m}\right)-\lim _{m} \lim _{p} u_{p}\left(h_{m}\right)\right| \leq \varepsilon
$$

for all sequences $\left(u_{p}\right)_{p} \subset B$ and $\left(h_{m}\right)_{m} \subset H$ provided the involved limits exist. For $\varepsilon=0$ we say $H$ interchanges limits with $B$. Fix $n \in \mathbb{N}$ and let $H$ be a bounded subset of a Fréchet space $E$. Denote

$$
\gamma_{n}(H):=\inf \left\{\varepsilon \geq 0: H \varepsilon \text {-interchanges limits with } U_{n}^{0}\right\} .
$$

We need the following two results from [6] and [7], respectively. 
Proposition 3.1. [6, Proposition 5] If $H$ is a bounded subset of a Fréchet space $E$ and $n \in \mathbb{N}$ then

$$
k_{n}(H) \leq \gamma_{n}(H) \leq 2 k_{n}(H) .
$$

In particular $H$ is weakly relatively compact if and only if $\gamma_{n}(H)=0$ for all $n \in \mathbb{N}$.

Lemma 3.2. [7, Lemma 3.2] Let $\left(I_{n}\right)$ be a sequence of pairwise disjoint finite nonempty sets and let $\mu_{n}$ be a probability measure on $\mathcal{P}\left(I_{n}\right)$ for each $n$. Let $\left(A_{k}\right)$ be a sequence of subsets of $I=\bigcup_{n \in \mathbb{N}} I_{n}$ such that, for some $\delta>0$, $\liminf _{n} \mu_{n}\left(A_{k} \cap I_{n}\right)>\delta$ holds for every $k \in \mathbb{N}$. Then there is a subsequence $\left(A_{k_{i}}\right)$ such that $\bigcap_{i \leq j} A_{k_{i}} \neq \emptyset$ for each $j \geq 1$.

We need also the following somewhat technical lemma. Its proof uses some ideas similar to those that applied in the proof of [7, Theorem 3.3].

Lemma 3.3. Fix $n \in \mathbb{N}$. If $H$ is a bounded subset of a Fréchet space $E$, then

$$
\gamma_{n}(H)=\gamma_{n}(\mathrm{coH}) \text {. }
$$

Proof. Choose sequences $\left(u_{p}\right) \subset U_{n}^{0}$ and $\left(h_{m}\right) \subset c o H$ such that the involved limits exist, and

$$
d=\lim _{m} \lim _{p} u_{p}\left(h_{m}\right)-\lim _{p} \lim _{m} u_{p}\left(h_{m}\right)>0 .
$$

Since $h_{m} \in c o H$, for each $m$ we have $h_{m}=\sum_{i \in I_{m}} t_{i} k_{i}$, where $k_{i} \in H$, and $I_{m}$ is a finite set, $0 \leq t_{i} \leq 1$ for all $i \in I_{m}$ and $\sum_{i \in I_{m}} t_{i}=1$. Without loss of generality we may assume that the sets $I_{m}$ are pairwise disjoint. Define $I=\bigcup_{m} I_{m}$. Since the set $H$ is bounded, there exists $M>0$ such that $\left|u_{p}\left(k_{i}\right)\right|<M$ for all $p, i \in \mathbb{N}$. Consequently, we may also assume that for each $i \in \mathbb{N}$, there is some $x_{i} \in[-M, M]$ such that

$$
\lim _{p} u_{p}\left(k_{i}\right)=x_{i} \text {. }
$$

For each $m \in \mathbb{N}$ define

$$
y_{m}=\lim _{p} u_{p}\left(h_{m}\right)=\lim _{p} \sum_{i \in I_{m}} t_{i} u_{p}\left(k_{i}\right)=\sum_{i \in I_{m}} t_{i} x_{i} .
$$

Then

$$
\begin{aligned}
d & =\lim _{m} \lim _{p} u_{p}\left(h_{m}\right)-\lim _{p} \lim _{m} u_{p}\left(h_{m}\right)=\lim _{m} y_{m}-\lim _{p} \lim _{m} u_{p}\left(h_{m}\right) \\
& =\lim _{p} \lim _{m}\left(y_{m}-u_{p}\left(h_{m}\right)\right) .
\end{aligned}
$$

Fix $\varepsilon>0$. We may assume that

$$
\lim _{m}\left(y_{m}-u_{p}\left(h_{m}\right)\right)>d-\varepsilon
$$

for every $p$. Then for each $p \in \mathbb{N}$ there exists $m_{p} \in \mathbb{N}$ such that if $m>m_{p}$, then

$$
y_{m}-u_{p}\left(h_{m}\right)>d-\varepsilon .
$$

For every $m \in \mathbb{N}$ define $\mu_{m}$, the probability measure on $I_{m}$, as

$$
\mu_{m}(A)=\sum_{i \in A} t_{i}
$$


Then we define

$$
A_{p}=\left\{i \in I: x_{i}-u_{p}\left(k_{i}\right)>d-2 \varepsilon\right\} .
$$

Observe that the following holds.

$$
\begin{aligned}
d-\varepsilon & \stackrel{(3.3)}{<} y_{m}-u_{p}\left(h_{m}\right) \stackrel{(3.2)}{=} \sum_{i \in I_{m}} t_{i} x_{i}-\sum_{i \in I_{m}} t_{i} u_{p}\left(k_{i}\right) \\
& =\sum_{i \in I_{m} \cap A_{p}} t_{i}\left(x_{i}-u_{p}\left(k_{i}\right)\right)+\sum_{i \in I_{m} \backslash A_{p}} t_{i}\left(x_{i}-u_{p}\left(k_{i}\right)\right) \\
& \stackrel{(3.4)}{\leq} \sum_{i \in I_{m} \cap A_{p}} t_{i} 2 M+d-2 \varepsilon=2 M \mu_{m}\left(I_{m} \cap A_{p}\right)+d-2 \varepsilon,
\end{aligned}
$$

so $\mu_{m}\left(I_{m} \cap A_{p}\right)>\varepsilon /(2 M)$, and then

$$
\liminf _{m} \mu_{m}\left(I_{m} \cap A_{p}\right) \geq \varepsilon /(2 M) .
$$

By Lemma 3.2 there exists a subsequence $\left(A_{p_{k}}\right)_{k}$ such that $\bigcap_{k \leq l} A_{p_{k}} \neq \emptyset$ for each $l \in \mathbb{N}$. So, by the definition of $A_{p}$, for each $l$ there exists an $i_{l}$ such that

$$
x_{i_{l}}-u_{p_{k}}\left(k_{i_{l}}\right)>d-2 \varepsilon
$$

for all $k \leq l$. Choosing subsequences we may assume that $\left(u_{p_{k}}\left(k_{i_{l}}\right)\right)_{l}$ converges to some $z_{k} \in \mathbb{R}$ for all $k$ and $\left(x_{i_{l}}\right)_{l}$ converges to some $x \in \mathbb{R}$. We may also assume that the sequence $\left(z_{k}\right)_{k}$ converges to some $z \in \mathbb{R}$. Then

$$
\lim _{l} \lim _{k} u_{p_{k}}\left(k_{i_{l}}\right) \stackrel{(3.1)}{=} \lim _{l} x_{i_{l}}=x
$$

and

$$
\lim _{k} \lim _{l} u_{p_{k}}\left(k_{i_{l}}\right)=\lim _{k} z_{k}=z .
$$

Inequality (3.5) implies that

$$
x-z_{k}=\lim _{l}\left(x_{i_{l}}-u_{p_{k}}\left(k_{i_{l}}\right)\right) \geq d-2 \varepsilon,
$$

so $x-z=\lim _{k}\left(x-z_{k}\right) \geq d-2 \varepsilon$. Then we derive that $\gamma_{n}(H) \geq d-2 \varepsilon$. Since $\varepsilon>0$ is arbitrary, we obtain that

$$
\gamma_{n}(H) \geq d=\lim _{m} \lim _{p} u_{p}\left(h_{m}\right)-\lim _{p} \lim _{m} u_{p}\left(h_{m}\right),
$$

and this holds for all sequences $\left(u_{p}\right) \subset U_{n}^{0}$ and $\left(h_{m}\right) \subset c o H$ such that the involved limits exist. Then we conclude that

$$
\gamma_{n}(H) \geq \gamma_{n}(\mathrm{coH}) \geq \gamma_{n}(H),
$$

and the proof is finished.

From Lemma 3.3 and Proposition 3.1 we obtain the following corollary.

Corollary 3.4. If $H$ is a bounded subset of a Fréchet space $E$ and $n \in \mathbb{N}$, then

$$
k_{n}(c o H) \leq 2 k_{n}(H) .
$$

Now we are ready to prove the main result of the paper. 
Theorem 3.5. If $H$ is a bounded subset of a Fréchet space $E$ and $n \in \mathbb{N}$, then

$$
k(c o H)<\left(2^{n+1}-2\right) k(H)+\frac{1}{2^{n}} .
$$

Proof. If $k(H)=0$ then $k_{n}(H)=0$ for all $n \in \mathbb{N}$, so by Corollary 3.4 we have $k(c o H)=0$ and inequality (3.7) holds. Thus, we may assume that $k(H)>0$. By Lemma 2.1 and Corollary 3.4, and applying that the function $f(x)=x /(1+x)$ is strictly increasing, we derive that

$$
k(\mathrm{coH}) \leq \frac{2^{n}-1}{2^{n}} \frac{k_{n}(\mathrm{coH})}{1+k_{n}(\mathrm{coH})}+\frac{1}{2^{n}} \leq \frac{2^{n}-1}{2^{n}} \frac{2 k_{n}(H)}{1+2 k_{n}(H)}+\frac{1}{2^{n}} .
$$

To finish the proof we need only to prove that

$$
\frac{1}{2^{n}} \frac{2 k_{n}(H)}{1+2 k_{n}(H)}<2 k(H) .
$$

If $k_{n}(H)=0$, inequality (3.9) holds indeed. Suppose that $k_{n}(H)>0$. Then

$$
\rho:=\sup _{h \in \bar{H}^{\sigma\left(E^{\prime \prime}, E^{\prime}\right)}} \inf _{e \in E} \frac{1}{2^{n}} \frac{d_{n}(h, e)}{1+d_{n}(h, e)}=\frac{1}{2^{n}} \frac{k_{n}(H)}{1+k_{n}(H)}>0,
$$

where to fix the last equality we applied the fact that the function $f(x)$ is strictly increasing. Fix $h_{0} \in \bar{H}^{\sigma\left(E^{\prime \prime}, E^{\prime}\right)}$ such that

$$
\alpha:=\inf _{e \in E} \frac{d_{n}\left(h_{0}, e\right)}{1+d_{n}\left(h_{0}, e\right)}>2^{n-1} \rho .
$$

Since $d_{m}(h, e) \leq d_{m+1}(h, e)$ for $m \in \mathbb{N}$ and $h, e \in E^{\prime \prime}$, we have

$$
\begin{aligned}
\inf _{e \in E} \sum_{m=n}^{\infty} \frac{1}{2^{m}} \frac{d_{m}\left(h_{0}, e\right)}{1+d_{m}\left(h_{0}, e\right)} & \geq \inf _{e \in E} \sum_{m=n}^{\infty} \frac{1}{2^{m}} \frac{d_{n}\left(h_{0}, e\right)}{1+d_{n}\left(h_{0}, e\right)}= \\
& =\sum_{m=n}^{\infty} \frac{1}{2^{m}} \alpha=\frac{1}{2^{n-1}} \alpha>\rho .
\end{aligned}
$$

Therefore

$$
k(H)=\sup _{h \in \bar{H}^{\sigma\left(E^{\prime \prime}, E^{\prime}\right)}} \inf _{e \in E} \sum_{m=1}^{\infty} \frac{1}{2^{m}} \frac{d_{m}(h, e)}{1+d_{m}(h, e)}>\rho .
$$

Consequently we note that

$$
\frac{1}{2^{n}} \frac{2 k_{n}(H)}{1+2 k_{n}(H)} \leq \frac{1}{2^{n}} \frac{2 k_{n}(H)}{1+k_{n}(H)}=2 \rho<2 k(H),
$$

so inequality (3.9) holds indeed and this completes the proof.

Corollary 3.6. If $H$ is a bounded set in a Fréchet space $E$ such that $k(H)>0$ then

$$
k(c o H)<\sqrt{k(H)}(3-2 \sqrt{k(H)}) .
$$


Proof. If $1 / 4<k(H) \leq 1$ the inequality (3.10) holds because

$$
\sqrt{k(H)}(3-2 \sqrt{k(H)}) \geq 1>k(c o H) .
$$

Observe that, since $\mathrm{coH}$ is a bounded set, the inequality $k(\mathrm{coH})<1$ holds. Therefore we may assume that $0<k(H) \leq 1 / 4$. Then there exists $n \in \mathbb{N}$ such that

By Theorem 3.5 we conclude that

$$
\frac{1}{4^{n+1}}<k(H) \leq \frac{1}{4^{n}}
$$

$$
k(c o H)<\left(2^{n+1}-2\right) k(H)+\frac{1}{2^{n}} .
$$

For each $n \in \mathbb{N}$ define two real functions

$$
f_{n}(x)=\left(2^{n+1}-2\right) x+\frac{1}{2^{n}} \text { and } g(x)=\sqrt{x}(3-2 \sqrt{x}) .
$$

Then

$$
f_{n}\left(1 / 4^{n}\right)=g\left(1 / 4^{n}\right), \quad f_{n}\left(1 / 4^{n+1}\right)=g\left(1 / 4^{n+1}\right) .
$$

Since $(g-f)^{\prime \prime}<0$ in $\left[1 / 4^{n+1}, 1 / 4^{n}\right]$, we deduce that $g(x) \geq f_{n}(x)$ in $\left[1 / 4^{n+1}, 1 / 4^{n}\right]$. Then by $(3.11)$ we conclude

$$
k(c o H)<f_{n}(k(H)) \leq g(k(H))=\sqrt{k(H)}(3-2 \sqrt{k(H)}) .
$$

Remark 3.7. If $k(H)=1 / 4^{n}$ for some $n \in \mathbb{N}$, then the upper bounds for $k(\mathrm{coH})$ from Theorem 3.5 and Corollary 3.6 are the same. Indeed, if we denote $f_{n}(x)=\left(2^{n+1}-2\right) x+\frac{1}{2^{n}}$, then $f_{n}(x) \leq f_{n+1}(x)$ if and only if $x \geq$ $1 / 4^{n+1}$, so

$$
\inf _{m \in \mathbb{N}} f_{m}(x)=f_{n}(x) \text { if } \frac{1}{4^{n+1}} \leq x \leq \frac{1}{4^{n}} .
$$

Then the upper bound for the function $k(\mathrm{coH})$ from Theorem 3.5 is $f_{n}\left(1 / 4^{n}\right)$, that is equal to $\sqrt{1 / 4^{n}}\left(3-2 \sqrt{1 / 4^{n}}\right)$.

Corollary 3.8 (Krein). If $H$ is a weakly relatively compact set in a Fréchet space, then $\mathrm{coH}$ is a weakly relatively compact set in $E$.

\section{Two additional measures of weak non-compactness $l k(H)$ and $k^{\prime}(H)$}

We start with the definition of next two measures of weak non-compactness for Fréchet spaces.

Definition 4.1. Let $H$ be a bounded subset of a Fréchet space $E$. Set

$$
l k(H):=\sup _{h \in \bar{H}^{\sigma\left(E^{\prime \prime}, E^{\prime}\right)}} \sum_{n=1}^{\infty} \frac{1}{2^{n}} \frac{d_{n}(h, E)}{1+d_{n}(h, E)}, \quad k^{\prime}(H):=\sum_{n=1}^{\infty} \frac{1}{2^{n}} \frac{k_{n}(H)}{1+k_{n}(H)} .
$$

The first observation shows the relation between new defined measures and the measure $k(H)$ and provides their equality for the case $E$ being a Banach space. 
Proposition 4.2. If $H$ is a bounded subset of a Fréchet space $E$ then $l k(H) \leq$ $k(H)$ and $l k(H) \leq k^{\prime}(H)$. If $E$ is a Banach space, and $U_{n}=U_{1}$ is the unit ball for all $n \in \mathbb{N}$, then $k^{\prime}(H)=k(H)=l k(H)$.

Proof. Since the function $f(x)=x /(1+x)$ is strictly increasing in $[0,+\infty)$, for a bounded set $A \subset[0,+\infty)$ we have

$$
\sup _{x \in A} f(x)=f\left(\sup _{x \in A} x\right) \text { and } \inf _{x \in A} f(x)=f\left(\inf _{x \in A} x\right) .
$$

Then

$$
\begin{aligned}
\sup _{h \in \bar{H}^{\sigma\left(E^{\prime \prime}, E^{\prime}\right)}} \sum_{n=1}^{\infty} \frac{1}{2^{n}} \frac{d_{n}(h, E)}{1+d_{n}(h, E)} & \leq \sum_{n=1}^{\infty} \sup _{h \in \bar{H}^{\sigma\left(E^{\prime \prime}, E^{\prime}\right)}} \frac{1}{2^{n}} \frac{d_{n}(h, E)}{1+d_{n}(h, E)}= \\
& \stackrel{(4.1)}{=} \sum_{n=1}^{\infty} \frac{1}{2^{n}} \frac{k_{n}(H)}{1+k_{n}(H)},
\end{aligned}
$$

so $l k(H) \leq k^{\prime}(H)$. Next we show that $l k(H) \leq k(H)$. For this observe that

$$
\begin{aligned}
d(h, E) & =\inf _{e \in E} \sum_{n=1}^{\infty} \frac{1}{2^{n}} \frac{d_{n}(h, e)}{1+d_{n}(h, e)} \geq \sum_{n=1}^{\infty} \inf _{e \in E} \frac{1}{2^{n}} \frac{d_{n}(h, e)}{1+d_{n}(h, e)} \stackrel{(4.1)}{=} \\
& =\sum_{n=1}^{\infty} \frac{1}{2^{n}} \frac{\inf _{e \in E} d_{n}(h, e)}{1+\inf _{e \in E} d_{n}(h, e)}=\sum_{n=1}^{\infty} \frac{1}{2^{n}} \frac{d_{n}(h, E)}{1+d_{n}(h, E)}
\end{aligned}
$$

Taking the supremums over all $h \in \bar{H}^{\sigma\left(E^{\prime \prime}, E^{\prime}\right)}$, we conclude that $l k(H) \leq$ $k(H)$. Finally, if $E$ is a Banach space and $U_{n}=U_{1}$ is the unit ball for all $n \in \mathbb{N}$, then $d_{n}=d_{m}$ and $k_{n}(H)=k_{m}(H)$ for all $n, m \in \mathbb{N}$. Hence

$$
\begin{aligned}
d(h, E) & =\inf _{e \in E} d(h, e)=\inf _{e \in E} \sum_{n=1}^{\infty} \frac{1}{2^{n}} \frac{d_{1}(h, e)}{1+d_{1}(h, e)} \\
& =\sum_{n=1}^{\infty} \inf _{e \in E} \frac{1}{2^{n}} \frac{d_{n}(h, e)}{1+d_{n}(h, e)} \stackrel{(4.1)}{=} \sum_{n=1}^{\infty} \frac{1}{2^{n}} \frac{d_{n}(h, E)}{1+d_{n}(h, E)}
\end{aligned}
$$

and then $k(H)$ equals to

$$
\begin{aligned}
\sup _{h \in \bar{H}^{\sigma\left(E^{\prime \prime}, E^{\prime}\right)}} \sum_{n=1}^{\infty} \frac{1}{2^{n}} \frac{d_{n}(h, E)}{1+d_{n}(h, E)} & =\sum_{n=1}^{\infty} \frac{1}{2^{n}} \sup _{h \in \bar{H}^{\sigma\left(E^{\prime \prime}, E^{\prime}\right)}} \frac{d_{1}(h, E)}{1+d_{1}(h, E)} \stackrel{(4.1)}{=} \\
& =\sum_{n=1}^{\infty} \frac{1}{2^{n}} \frac{k_{n}(H)}{1+k_{n}(H)} .
\end{aligned}
$$

This consequently yields the promised equalities $k(H)=l k(H)=k^{\prime}(H)$. The proof is completed.

For $x^{* *} \in E^{\prime \prime}$ we have $d\left(x^{* *}, E\right)=0$ if and only if $x^{* *} \in E$ if and only if $d_{n}\left(x^{* *}, E\right)=0$ for $n \in \mathbb{N}$. This provides the following relations.

Proposition 4.3. For a bounded subset $H$ of a Fréchet space $E$ the set $H$ is weakly relatively compact if and only if $k(H)=0$ if and only if $l k(H)=0$ if and only if $k^{\prime}(H)=0$ if and only if $k_{n}(H)=0$ for all $n \in \mathbb{N}$. 
Note also the following relations between functions $k(H)$ and $k^{\prime}(H)$ with $l k(H)$.

Proposition 4.4. If $H$ is a bounded subset of a Fréchet space $E$ and $n \in \mathbb{N}$ then

$$
k(H) \leq\left(2^{n}-1\right) \cdot l k(H)+\frac{1}{2^{n}} .
$$

Proof. The function $f(x)=x /(1+x)$ is strictly increasing and $d_{m}(h, e) \leq$ $d_{n}(h, e)$ for each $h, e \in E^{\prime \prime}$ and $m \leq n$, so we derive that

$$
\begin{aligned}
\sum_{m=1}^{\infty} \frac{1}{2^{m}} \frac{d_{m}(h, e)}{1+d_{m}(h, e)} & \leq \sum_{m=1}^{n} \frac{1}{2^{m}} \frac{d_{n}(h, e)}{1+d_{n}(h, e)}+\sum_{m=n+1}^{\infty} \frac{1}{2^{m}} \\
& \leq\left(1-\frac{1}{2^{n}}\right) \frac{d_{n}(h, e)}{1+d_{n}(h, e)}+\frac{1}{2^{n}}= \\
& =\left(2^{n}-1\right) \frac{1}{2^{n}} \cdot \frac{d_{n}(h, e)}{1+d_{n}(h, e)}+\frac{1}{2^{n}}
\end{aligned}
$$

Then

$$
\begin{aligned}
d(h, E) & =\inf _{e \in E} \sum_{m=1}^{\infty} \frac{1}{2^{m}} \cdot \frac{d_{m}(h, e)}{1+d_{m}(h, e)} \leq\left(2^{n}-1\right) \frac{1}{2^{n}} \frac{\inf _{e \in E} d_{n}(h, e)}{1+\inf _{e \in E} d_{n}(h, e)}+\frac{1}{2^{n}} \leq \\
& \leq\left(2^{n}-1\right) \sum_{m=1}^{\infty} \frac{1}{2^{m}} \cdot \frac{d_{m}(h, E)}{1+d_{m}(h, E)}+\frac{1}{2^{n}} .
\end{aligned}
$$

This yields the following inequality when the supremum is taken over all $h \in \bar{H}^{\sigma\left(E^{\prime \prime}, E^{\prime}\right)}$.

$$
k(H) \leq\left(2^{n}-1\right) \sup _{h \in \bar{H}^{\sigma\left(E^{\prime \prime}, E^{\prime}\right)}} \sum_{m=1}^{\infty} \frac{1}{2^{m}} \cdot \frac{d_{m}(h, E)}{1+d_{m}(h, E)}+\frac{1}{2^{n}}=\left(2^{n}-1\right) l k(H)+\frac{1}{2^{n}} .
$$

The proof is completed.

Proposition 4.5. If $H$ is a bounded subset of a Fréchet space $E$ and $n \in \mathbb{N}$ then

$$
k^{\prime}(H)<n \cdot l k(H)+\frac{1}{2^{n}} .
$$

Proof. Fix $m \in \mathbb{N}$. Then

$$
\frac{1}{2^{m}} \frac{k_{m}(H)}{1+k_{m}(H)}=\sup _{h \in \bar{H}^{\sigma\left(E^{\prime \prime}, E^{\prime}\right)}} \frac{1}{2^{m}} \frac{d_{m}(h, E)}{1+d_{m}(h, E)} \leq l k(H)
$$


Since $H$ is a bounded set, we note the following $k_{m}(H)<+\infty$ for all $m \in \mathbb{N}$. Consequently we have

$$
\begin{aligned}
k^{\prime}(H) & =\sum_{m=1}^{n} \frac{1}{2^{m}} \frac{k_{m}(H)}{1+k_{m}(H)}+\sum_{m=n+1}^{\infty} \frac{1}{2^{m}} \frac{k_{m}(H)}{1+k_{m}(H)} \stackrel{(4.4)}{<} \\
& <\sum_{m=1}^{n} l k(H)+\sum_{m=n+1}^{\infty} \frac{1}{2^{m}}=n \cdot l k(H)+\frac{1}{2^{n}}
\end{aligned}
$$

and the proof is finished.

The following proposition is a consequence of Proposition 3.1 and Lemma 3.3.

Proposition 4.6. Let $H$ be a bounded set in a Fréchet space $E$. Then $k^{\prime}(c o H) \leq$ $2 \cdot k^{\prime}(H)$.

We complete the paper with the following quantitative versions of Krein's theorem for the function $l k(H)$. First we prove the following

Proposition 4.7. If $H$ is a bounded set in a Fréchet space then

$$
l k(c o H)<2 n \cdot l k(H)+\frac{1}{2^{n-1}}
$$

for all $n \in \mathbb{N}$.

Proof. By Proposition 4.2, Proposition 4.6 and Proposition 4.5 we conclude that

$$
l k(c o H) \leq k^{\prime}(c o H) \leq 2 k^{\prime}(H)<2\left(n \cdot l k(H)+\frac{1}{2^{n}}\right)=2 n \cdot l k(H)+\frac{1}{2^{n-1}} .
$$

Corollary 4.8. If $H$ is a bounded set in a Fréchet space such that $l k(H)>0$ then

$$
l k(c o H)<\left(2 \log _{1 / 2} l k(H)+2\right) l k(H) .
$$

Proof. If $1 / 2<l k(H) \leq 1$, the inequality holds because then

$$
\left(2 \log _{1 / 2} l k(H)+2\right) l k(H) \geq 1>l k(c o H) .
$$

Therefore we can assume that $0<l k(H) \leq 1 / 2$. Then there exists $n \in \mathbb{N}$ such that

$$
\frac{1}{2^{n+1}}<l k(H) \leq \frac{1}{2^{n}}
$$

By Proposition 4.7 we conclude that

$$
l k(c o H)<2 n \cdot l k(H)+\frac{1}{2^{n-1}} .
$$

Let $f_{n}(x)=2 n x+\frac{1}{2^{n-1}}$ and $g(x)=\left(2 \log _{1 / 2} x+2\right) x$ for each $n \in \mathbb{N}$. Then

$$
f_{n}\left(1 / 2^{n}\right)=g\left(1 / 2^{n}\right), \quad f_{n}\left(1 / 2^{n+1}\right)=g\left(1 / 2^{n+1}\right) .
$$


Since $(g-f)^{\prime \prime}<0$ in $\left[1 / 2^{n+1}, 1 / 2^{n}\right]$, we deduce that $g(x) \geq f_{n}(x)$ in $\left[1 / 2^{n+1}, 1 / 2^{n}\right]$ for each $n \in \mathbb{N}$. Then, by inequality (4.5) we have

$$
l k(c o H)<f_{n}(l k(H)) \leq g(l k(H))=\left(2 \log _{1 / 2} l k(H)+2\right) l k(H) .
$$

Remark 4.9. If $l k(H)=1 / 2^{n}$ for some $n \in \mathbb{N}$, then the upper bounds for $l k(\mathrm{coH})$ from Proposition 4.7 and Corollary 4.8 are the same. Indeed, if we denote $f_{n}(x)=2 n x+\frac{1}{2^{n-1}}$, then $f_{n}(x) \leq f_{n+1}(x)$ if and only if $x \geq 1 / 2^{n+1}$, so

$$
\inf _{m \in \mathbb{N}} f_{m}(x)=f_{n}(x) \text { if } \frac{1}{2^{n+1}} \leq x \leq \frac{1}{2^{n}} .
$$

Then the upper bound for the function $l k(c o H)$ from Proposition 4.7 is $f_{n}\left(1 / 2^{n}\right)$, that is equal to $\left(2 \log _{1 / 2} 1 / 2^{n}+2\right) 1 / 2^{n}$.

We have also the following

Proposition 4.10. If $H$ is a bounded set in a Fréchet space then

$$
l k(c o H) \leq k(c o H)<\left(2^{n+1}-2\right) l k(H)+\frac{1}{2^{n}} .
$$

for all $n \in \mathbb{N}$.

Proof. By Proposition 4.2, Lemma 2.1 and Corollary 3.4 we conclude that

$$
\begin{aligned}
l k(c o H) & \leq k(c o H) \leq \frac{2^{n}-1}{2^{n}} \frac{k_{n}(c o H)}{1+k_{n}(c o H)}+\frac{1}{2^{n}} \leq \\
& \leq \frac{2^{n}-1}{2^{n}} \frac{2 k_{n}(H)}{1+2 k_{n}(H)}+\frac{1}{2^{n}}<\left(2^{n+1}-2\right) l k(H)+\frac{1}{2^{n}},
\end{aligned}
$$

where the proof of the last inequality is very similar to the proof of (3.9).

Using the same proof as in Corollary 3.6 we deduce the following corollary that provides a better bound for $l k(\mathrm{coH})$ for the case when $l k(H)>\frac{1}{16}$.

Corollary 4.11. If $H$ is a bounded set in a Fréchet space and $l k(H)>0$ then

$$
l k(c o H) \leq k(c o H)<\sqrt{l k(H)}(3-2 \sqrt{l k(H)}) .
$$

\section{References}

[1] C. Angosto, B. Cascales, Measures of weak noncompactness in Banach spaces, Topology Appl. 156 (2009), 1412-1421.

[2] C. Angosto, Distance to spaces of functions, $\mathrm{PhD}$ thesis, Universidad de Murcia (2007).

[3] C. Angosto, B. Cascales, A new look at compactness via distances to functions spaces, World Sc. Pub. Co. (2008).

[4] C. Angosto, B. Cascales, The quantitative difference between countable compactness and compactness J. Math. Anal. Appl. 343 (2008), 479-491.

[5] C. Angosto, B. Cascales, I. Namioka, Distances to spaces of Baire one functions, Math. Z. 263 (2009), 103-124. 
[6] C. Angosto, J. Kąkol and M. López-Pellicer, A quantitative approach to weak compactnesss in Fréchet spaces and spaces $C(X)$. Preprint.

[7] B. Cascales, W. Marciszesky and M. Raja. Distance to spaces of continuous functions. Topology Appl. 153 (2006), 2303-2319.

[8] M. Fabian, P. Habala, P. Hajek, V. Montesinos, J. Pelant, V. Zizler, Functional Analysis and Infinite-dimensional geometry, CMS Books in Mathematics, Canadian Math. Soc., Springer (2001).

[9] M. Fabian, P. Hájek, V. Montesinos, and V. Zizler, A quantitative version of Krein's theorem, Rev. Mat. Iberoam. 21 (2005), 237-248.

[10] A. S. Granero, An extension of the Krein-Smulian Theorem, Rev. Mat. Iberoam. 22 (2006), 93-110.

[11] A. S. Granero, P. Hájek, V. Montesinos Santalucía, Convexity and $\omega^{*}$ compactness in Banach spaces, Math. Ann. 328 (2004), 625-631.

[12] A. Grothendieck. Criteres de compacité dans les spaces fonctionnelles généraux. Amer. J. Math. 74 (1952), 168-186.

[13] S. S. Khurana, Weakly compactly generated Fréchet spaces, Int. J. Math. Math. Sci. 2 (1979), 721-724.

Carlos Angosto

Depto. de Matemática Aplicada y Estadistica: Universidad Politécnica de Cartagena, 30203 Cartagena, Spain

e-mail: angosto@um.es

Jerzy Ka̧kol

Faculty of Mathematics and Informatics A. Mickiewicz University 61-614 Poznań, Poland

e-mail: kakol@amu.edu.pl

Albert Kubzdela

Institute of Civil Engineering, Poznań University of Technology, Ul. Piotrowo 5, 61-138 Poznań, Poland

e-mail: albert.kubzdela@put.poznan.pl

Manuel López-Pellicer

Depto. de Matemática Aplicada and IUMPA. Universitat Politècnica de València, E-46022 Valencia, Spain

e-mail: mlopezpe@mat.upv.es 